\title{
TESTE DE ENVELHECIMENTO ACELERADO PARA AVALIAÇÃO DO POTENCIAL FISIOLÓGICO DE SEMENTES DE GRAMA-BERMUDA ${ }^{1}$
}

\author{
CLÍSSIA BARBOZA DA SILVA², KATHIA FERNANDES LOPES PIVETTA², \\ CÉSAR AUGUSTO VICTORINO MELO DE OLIVEIRA ${ }^{4}$, MARILSA APARECIDA RODRIGUES 5 , \\ ROBERVAL DAITON VIEIRA ${ }^{6}$
}

\begin{abstract}
RESUMO - A utilização de sementes para formação de gramados esportivos e ornamentais tem assumido grande importância no Brasil, principalmente pelo baixo custo em relação à formação por placas de tapetes naturais. As "bermudas" [Cynodon dactylon (L.) Pers] são as principais gramas utilizadas na formação de campos esportivos e, dentre os problemas enfrentados, destacase a dificuldade de estabelecimento adequado da cultura, fato que exige a utilização de sementes de alto potencial fisiológico, de modo a permitir rápida emergência e desenvolvimento das plantas. Assim, estudaram-se procedimentos para a condução do teste de envelhecimento acelerado para determinar o potencial fisiológico de sementes dessa espécie, incluindo a avaliação da eficiência do uso de solução saturada de $\mathrm{NaCl}$ como alternativa para a realização do teste. Para tanto, cinco lotes de sementes foram submetidos aos testes de germinação, emergência de plântulas e envelhecimento acelerado (períodos de 48,72 e 96 h, a 41 e $45^{\circ} \mathrm{C}$, com e sem o uso de solução saturada de $\mathrm{NaCl}$ ). $\mathrm{O}$ experimento foi conduzido em delineamento inteiramente casualizado. $\mathrm{O}$ envelhecimento acelerado com o uso de solução saturada de $\mathrm{NaCl}$, dentre os procedimentos estudados, é o método mais adequado para avaliação do potencial fisiológico de sementes de grama-bermuda, sendo que a combinação $45^{\circ} \mathrm{C} / 48 \mathrm{~h}$ é eficiente para a classificação dos lotes em diferentes níveis de vigor.
\end{abstract}

Termos para indexação: Cynodon dactylon, gramínea, vigor, germinação, solução saturada de $\mathrm{NaCl}$.

\section{ACCELERATED AGEING TEST TO EVALUATE THE PHYSIOLOGICAL POTENTIAL OF BERMUDA GRASS SEEDS}

\begin{abstract}
Seeds for forming ornamental and sports lawns has been very important in Brazil, mainly because of the lower cost compared to formatting lawns using plaques of natural carpets. Bermuda grass, [Cynodon dactylon (L.) Pers], is the main grass used on sports fields but one of the problems is establishing a suitable stand, a task that requires high quality seeds for rapid seedling emergence and initial plant development. The objective of this research was to study procedures for the accelerated ageing test to determine the physiological potential of seeds of this species, including the evaluation of the efficiency of using saturated salt solution as an
\end{abstract}

${ }^{1}$ Submetido em 05/09/2009. Aceito para publicação em 08/12/2009.

${ }^{2}$ Eng. Agr., Mestranda em Produção Vegetal. FCAV/UNESP, 14884-900 - Jaboticabal, SP, clissia unesp@yahoo.com.br.

${ }^{3}$ Eng. Agr., Dra., Professora Assistente, Departamento de Produção Vegetal. FCAV/UNESP, 14884-900 - Jaboticabal, SP, kathia@fcav.unesp.br.

${ }^{4}$ Eng. Agr., Mestrando em Produção Vegetal. FCAV/UNESP, 14884-900
- Jaboticabal, SP, cavmo77@yahoo.com.br.

${ }^{5}$ Eng. Agr., Mestranda em Ciência do Solo. FCAV/UNESP, 14884-900 - Jaboticabal, SP, aprog@ig.com.br.

${ }^{6}$ Eng. Agr., Dr., Professor Titular, Departamento de Produção Vegetal. FCAV/UNESP, 14884-900 - Jaboticabal, SP, rdvieira@fcav.unesp.br. 
alternative to doing the test. Five seed lots were tested for germination, first count of germination, seedling emergence and accelerated ageing (periods of 48,72 and $96 \mathrm{~h}$ at 41 and $45{ }^{\circ} \mathrm{C}$, with and without the use of a saturated solution of $\mathrm{NaCl}$ ). The experiment was conducted using a completely randomized design. Among the procedures evaluated, the saturated salt accelerated ageing method (SSAA) is the most suitable for assessing the physiological potential of bermuda grass seeds. The combination $45^{\circ} \mathrm{C} / 48 \mathrm{~h}$ is the most efficient for classifying seed lots according to vigor.

Index terms: Cynodon dactylon, Gramineae, vigor, germination, saturated solution of $\mathrm{NaCl}$.

\section{INTRODUÇÃO}

O gramado constitui componente básico para o desenvolvimento paisagístico e esportivo. As chamadas "bermudas", também conhecidas como grama-seda [Cynodon dactylon (L.) Pers] são as mais indicadas para a formação de gramados esportivos, por apresentarem folhas macias e resistentes ao pisoteio, além de ótima capacidade de regeneração no caso de injúrias (Arruda e Henriques, 1995).

A utilização de sementes para formação de novos gramados é prática comum em regiões como Europa e Estados Unidos, e atualmente vem crescendo consideravelmente no Brasil, principalmente pelo baixo custo em relação à formação de gramados por placas de tapetes de grama natural, tendo a utilização de semente de Cynodon dactylon crescido expressivamente.

No Brasil, poucos relatos existem com relação à germinação e vigor de sementes de espécies usadas em gramados esportivos e ornamentais; e sabe-se que a obtenção de sementes de alto potencial fisiológico é fundamental para o sucesso da produção agrícola, havendo, portanto, necessidade do desenvolvimento de testes que possam estimar o vigor, possibilitando a seleção dos melhores lotes para a comercialização, fornecendo com maior precisão, informações para a semeadura.

Os testes de vigor têm se constituído em ferramenta de uso cada vez mais rotineiro pelas indústrias de sementes para a determinação do potencial fisiológico. As empresas produtoras e as instituições oficiais têm incluído esses testes em programas internos de controle de qualidade ou para garantia da qualidade das sementes destinadas à comercialização (Marcos Filho, 1999a).

Neste sentido, pode-se destacar o teste de envelhecimento acelerado, que tem como princípio o fato da taxa de deterioração da semente ser aumentada consideravelmente pela exposição em níveis elevados de temperatura e umidade relativa do ar, considerados os fatores ambientais preponderantes na intensidade e velocidade de deterioração (Delouche, 2002). Dessa maneira, lotes mais vigorosos geralmente são menos afetados na capacidade de produzir plântulas normais e apresentam germinação mais elevada, após serem submetidos ao envelhecimento (Delouche e Baskin, 1973).

Para sementes de gramíneas, o teste de envelhecimento acelerado pode apresentar certas limitações, devido ao tamanho pequeno, apresentando rápida absorção de água, podendo levar a grande variação no teor de água após o envelhecimento, resultando em grau de deterioração mais acentuado e redução mais drástica da germinação (Powell, 1995; Panobianco e Marcos Filho, 1998; Spinola et al., 1998). Assim, têm sido estudados procedimentos alternativos para a condução deste teste, visando o controle da absorção de água pela semente durante a realização do teste, dentre estes, o uso de solução saturada de $\mathrm{NaCl}$, que permite a obtenção de umidades relativas inferiores às verificadas no envelhecimento acelerado convencional, pela absorção de água de forma mais lenta e em menor intensidade, culminando com menor grau de deterioração (Jianhua e McDonald, 1996).

Desta forma, este trabalho foi desenvolvido com o objetivo de estudar a metodologia do teste de envelhecimento acelerado para avaliação do potencial fisiológico de sementes de grama-bermuda, bem como avaliar a eficiência do uso de solução saturada de $\mathrm{NaCl}$ como alternativa para a realização do teste.

\section{MATERIAL É MÉTODOS}

O experimento foi conduzido no Laboratório de Análise de Sementes do Departamento de Produção Vegetal da UNESP/FCAV, Campus de Jaboticabal, SP. Foram utilizados cinco lotes de sementes de grama-bermuda, os quais foram submetidos aos testes e, ou determinações descritas a seguir:

Teor de água - determinado com duas subamostras de $4 \mathrm{~g}$ de sementes para cada lote, pelo método de estufa a $105 \pm 3{ }^{\circ} \mathrm{C}$, durante 24 horas, de acordo com as Regras para Análise de Sementes (Brasil, 1992). Essa determinação foi realizada antes e após os períodos de envelhecimento, visando avaliação da uniformidade das condições do teste. 
Germinação - conduzido com quatro repetições de 100 sementes para cada lote, semeadas em caixas de plástico (11 x 11 x 3,5 cm) tipo "gerbox", sobre papel de filtro umedecido com água destilada na proporção de 2,5 vezes o seu peso seco, mantidas em câmara de germinação regulada à temperatura de $20-30{ }^{\circ} \mathrm{C}$, com fotoperíodo de oito horas. As avaliações foram feitas diariamente, durante 21 dias, computando-se a percentagem de plântulas normais (Brasil, 1992).

Primeira contagem da germinação - constou da determinação da percentagem de plântulas normais aos sete dias após a semeadura por ocasião da realização do teste de germinação.

Emergência de plântulas - foi conduzido em viveiro experimental de plantas ornamentais e florestais do Campus. Utilizaram-se quatro repetições de 100 sementes, distribuídas em bandejas de plástico contendo terra e areia na proporção de $1: 1$, em sulcos longitudinais de $0,5 \mathrm{~cm}$ de profundidade distanciados cinco centímetros entre si. Avaliaram-se a velocidade de emergência de plântulas, mediante a contagem diária de plântulas emergidas, e a percentagem de emergência de plântulas aos 21 dias após a semeadura. O cálculo do índice de velocidade foi realizado de acordo com Maguire (1962).

Envelhecimento acelerado tradicional - foram utilizadas caixas de plástico transparentes $(11 \times 11 \times 3,5 \mathrm{~cm})$, como compartimento individual (mini-câmaras), contendo em seu interior tela metálica acoplada e uma tela de nylon sobreposta para dar suporte às sementes. Dentro das caixas foram colocados $40 \mathrm{~mL}$ de água destilada, para a obtenção de aproximadamente $100 \%$ de umidade relativa do ar. As caixas foram tampadas e mantidas no interior de câmaras de envelhecimento, a 41 e $45^{\circ} \mathrm{C}$, durante 48 , 72 e 96 horas. Decorrido cada período de envelhecimento, as sementes foram colocadas para germinar seguindo metodologia descrita para o teste de germinação. A avaliação da percentagem de plântulas normais foi realizada no sétimo dia após a semeadura.

Envelhecimento acelerado com solução saturada de NaCl (EASS) - conduzido de forma semelhante à descrita para o procedimento tradicional, substituindo-se os $40 \mathrm{~mL}$ de água destilada por igual quantidade de solução saturada de $\mathrm{NaCl}$ (40 g/100 mL de água), estabelecendo ambiente com $76 \%$ de umidade relativa do ar, segundo metodologia descrita por Jianhua e McDonald (1996).

Foi utilizado o delineamento experimental inteiramente casualizado. Os dados foram submetidos à análise de variância, sendo que, os de percentagem de germinação foram previamente transformados em arc sen $(x / 100)^{1 / 2}$. As médias foram comparadas pelo teste de Scott-Knott a 5\% de probabilidade de erro.

\section{RESULTADOS E DISCUSSÃO}

Os teores iniciais de água dos cinco lotes de sementes de grama-bermuda variaram de 7,3 a 7,9\% (Tabela 1). Essa pequena variação proporciona segurança na execução dos testes, considerando-se que a uniformização do teor de água das sementes é imprescindível para a padronização das avaliações e obtenção de resultados consistentes (Torres e Marcos Filho, 2001).

TABELA 1. Teor de água (\%) de sementes de grama-bermuda (Cynodon dactylon) antes e após envelhecimento acelerado tradicional (EAT) e com solução saturada de $\mathrm{NaCl}$ (EASS), a $41{ }^{\circ} \mathrm{C}$ e $45{ }^{\circ} \mathrm{C}$, em diferentes períodos de tempo.

\begin{tabular}{cccccc}
\hline \multirow{2}{*}{ Tratamentos } & \multicolumn{5}{c}{ Lote } \\
\cline { 2 - 6 } & 1 & 2 & 3 & 4 & 5 \\
\hline Inicial & 7,4 & 7,3 & 7,6 & 7,7 & 79 \\
\hline $41^{\circ} \mathrm{C} /$ EAT & & & & & \\
$48 \mathrm{~h}$ & 41,8 & 41,5 & 42,8 & 40,4 & 41,4 \\
$72 \mathrm{~h}$ & 42,8 & 43,6 & 45,2 & 44,0 & 42,8 \\
$96 \mathrm{~h}$ & 46,0 & 46,1 & 49,2 & 45,3 & 43,5 \\
\hline $41^{\circ} \mathrm{C} / \mathrm{EASS}$ & & & & & \\
$48 \mathrm{~h}$ & 8,1 & 8,4 & 10,1 & 9,4 & 8,3 \\
$72 \mathrm{~h}$ & 8,1 & 8,2 & 10,3 & 10,1 & 8,8 \\
$96 \mathrm{~h}$ & 9,9 & 10,0 & 11,1 & 10,5 & 10,9 \\
\hline $45^{\circ} \mathrm{C} / \mathrm{EAT}$ & & & & & \\
$48 \mathrm{~h}$ & 37,7 & 43,2 & 41,9 & 41,7 & 44,0 \\
$72 \mathrm{~h}$ & 42,9 & 42,3 & 45,9 & 43,6 & 41,9 \\
$96 \mathrm{~h}$ & 45,6 & 46,7 & 44,7 & 44,6 & 44,4 \\
\hline $45^{\circ} \mathrm{C} / \mathrm{EASS}$ & & & & & \\
$48 \mathrm{~h}$ & 8,4 & 8,0 & 7,9 & 8,2 & 8,1 \\
$72 \mathrm{~h}$ & 8,2 & 9,4 & 8,5 & 10,8 & 9,4 \\
$96 \mathrm{~h}$ & 9,9 & 9,3 & 9,9 & 11,0 & 10,6 \\
\hline
\end{tabular}

Após a exposição ao envelhecimento acelerado tradicional e com o uso de solução saturada de $\mathrm{NaCl}$ (Tabela 1), verificou-se que, independentemente do período de exposição e temperatura associada, o emprego de solução saturada de $\mathrm{NaCl}$, resultou em teores de água acentuadamente inferiores e mais uniformes que os observados para as sementes envelhecidas pelo procedimento tradicional. Considerando- 
se que o teor de água da semente depende da umidade relativa do ar (Carvalho e Nakagawa, 2000), estes resultados estão de acordo com as informações de Jianhua e McDonald (1996), indicando que o uso de solução salina acarreta menor velocidade de absorção de água pela semente, permitindo a obtenção de teores de água relativamente inferiores aos verificados no envelhecimento tradicional (100\% U.R.), sem reduzir a eficiência do teste. Resultados semelhantes foram observados em aveia preta (Souza et al., 2009).

No envelhecimento acelerado tradicional, as diferenças de teores deágua entre os lotes variaram entre 2,3 a $6,3 \%$ pontos percentuais, dependendo da combinação temperatura/período de exposição (Tabela 1). Já para as sementes submetidas ao envelhecimento acelerado modificado, essa variação passou a ser de 0,5 a $2,6 \%$ pontos percentuais, dentro dos limites considerados toleráveis, que é de 3 a $4 \%$ (Marcos Filho, 1999b). Considerando as combinações temperatura/período de exposição, observou-se que, de modo geral, a combinação $45{ }^{\circ} \mathrm{C} / 48 \mathrm{~h}$, pelo método modificado, proporcionou maior redução na velocidade e maior uniformidade no processo de absorção de água pelas sementes.

A utilização de solução saturada de sal contribuiu também para reduzir a microflora fúngica durante a condução do teste, em função da restrição da umidade relativa do ambiente no interior das caixas plásticas que desfavoreceu a proliferação de fungos. Constatações semelhantes foram efetuadas por Rodo et al. (2000) em sementes de cenoura, Ramos et al. (2004) em rúcula, Ávila et al. (2006) em rabanete e Souza et al. (2009) em aveia preta.

Os resultados relativos ao potencial fisiológico inicial dos cinco lotes de sementes de grama-bermuda estão apresentados na Tabela 2. Os valores obtidos no teste de germinação não permitiram diferenciar os lotes de sementes de grama-bermuda; por essa razão, os lotes foram comparados quanto ao vigor, para obtenção de informações mais detalhadas. Os resultados obtidos na primeira contagem de germinação permitiram a separação dos lotes em duas classes, apontando os lotes 1, 3 e 4 como de desempenho superior e os lotes 2 e 5 de padrão inferior. O índice de velocidade de emergência de plântulas apresentou baixa sensibilidade na classificação dos lotes em relação ao vigor, conseguindo destacar apenas o lote 1 como o mais vigoroso. Por outro lado, evidenciou-se a separação dos lotes quanto à percentagem de emergência de plântulas, sendo os lotes 1 e 3 considerados de alto vigor e, os lotes 2, 4 e 5, de baixo vigor. Dessa forma, verificaram-se respostas diferenciadas dos lotes quando submetidas às diferentes situações específicas de cada teste, o que destaca a importância da utilização de vários testes para avaliação do vigor de sementes.
TABELA 2. Germinação (G), primeira contagem de germinação (PC), emergência de plântulas (EP) e índice de velocidade de emergência (IVE) de cinco lotes de sementes de grama-bermuda (Cynodon dactylon) ${ }^{1}$.

\begin{tabular}{ccccc}
\hline Lote & $\mathrm{G}^{2}$ & $\mathrm{PC}^{2}$ & $\mathrm{IVE}$ & $\mathrm{EP}^{2}$ \\
\hline 1 & $79 \mathrm{a}$ & $63 \mathrm{a}$ & $15,53 \mathrm{a}$ & $70 \mathrm{a}$ \\
2 & $76 \mathrm{a}$ & $48 \mathrm{~b}$ & $12,20 \mathrm{~b}$ & $57 \mathrm{~b}$ \\
3 & $74 \mathrm{a}$ & $59 \mathrm{a}$ & $11,34 \mathrm{~b}$ & $69 \mathrm{a}$ \\
4 & $81 \mathrm{a}$ & $59 \mathrm{a}$ & $11,58 \mathrm{~b}$ & $57 \mathrm{~b}$ \\
5 & $75 \mathrm{a}$ & $46 \mathrm{~b}$ & $11,58 \mathrm{~b}$ & $61 \mathrm{~b}$ \\
\hline $\mathrm{CV}(\%)$ & 8,8 & 10,6 & 9,2 & 6,4 \\
\hline
\end{tabular}

${ }^{1}$ Médias seguidas pela mesma letra, na coluna, não diferem entre si pelo teste de Scott-Knott a 5\% de probabilidade.

${ }^{2}$ Dados transformados em $\operatorname{arc} \operatorname{sen}(\mathrm{x} / 100)^{1 / 2}$

O teste de envelhecimento acelerado conduzido a $41{ }^{\circ} \mathrm{C}$ pelo método tradicional (Tabela 3 ), não permitiu o ranqueamento consistente dos lotes em níveis de vigor, talvez devido aos teores relativamente elevados de água atingidos pelas sementes durante o teste, resultando em maior grau de deterioração e, consequentemente, resultados desuniformes. A exposição durante 48 horas revelou divergência dos resultados quando comparados à primeira contagem de germinação, índice de velocidade e percentagem de emergência das plântulas, ao posicionar isoladamente o lote 4 no grupo de alto potencial e os demais lotes no grupo de baixo potencial. No período de 72 horas de envelhecimento, houve menor homogeneidade dos resultados e aumento no coeficiente de variação, não permitindo a separação dos lotes em níveis de vigor. O estresse provocado por 96 horas de exposição, também se mostrou pouco promissor, ao posicionar os lotes 1 e 2 no grupo de baixo potencial e os lotes 3 , 4 e 5 no grupo de alto potencial. $\mathrm{O}$ envelhecimento acelerado tradicional tem revelado resultados pouco reproduzíveis para espécies de sementes pequenas, uma vez que estas absorvem água mais rapidamente, resultando em teores de água elevados e deterioração mais acentuada (Powell, 1995; Panobianco e Marcos Filho, 1998).

Quando se utilizou solução saturada de $\mathrm{NaCl}$, a $41^{\circ} \mathrm{C}$, os períodos de 48 e 72 horas de exposição mostraram estreita relação com os resultados do teste de emergência de plântulas, ao enquadrar os lotes 1 e 3 dentro do grupo de alto vigor e os lotes 2,4 e 5 no grupo de baixo vigor (Tabela 3). Resultados semelhantes foram observados por Torres e Marcos Filho 
(2001), trabalhando com sementes de maxixe utilizando $41{ }^{\circ} \mathrm{C} / 48$ h, quanto à comparação com os resultados de emergência de plântulas, normalmente utilizado como referência para os resultados dos testes de vigor (Marcos Filho, 2005). Já o período de exposição de 96 horas, apresentou resultados pouco consistentes, ao designar os lotes 1,2 e 5 de alta qualidade e os lotes 3 e 4 de baixa qualidade.

TABELA 3. Vigor de cinco lotes de sementes de grama-bermuda (Cynodon dactylon) avaliado pelo teste de envelhecimento acelerado tradicional (EAT) e com uso de solução saturada de $\mathrm{NaCl}$ (EASS), a $41{ }^{\circ} \mathrm{C}$, em três períodos de envelhecimento ${ }^{1,2}$.

\begin{tabular}{cccccccc}
\hline \multirow{2}{*}{ Lote } & \multicolumn{3}{c}{ EAT } & & \multicolumn{3}{c}{$\mathrm{NaCl}$} \\
\cline { 2 - 4 } \cline { 7 - 8 } \cline { 7 - 8 } & $48 \mathrm{~h}$ & $72 \mathrm{~h}$ & $96 \mathrm{~h}$ & & $48 \mathrm{~h}$ & $72 \mathrm{~h}$ & $96 \mathrm{~h}$ \\
\hline 1 & $22 \mathrm{~b}$ & $21 \mathrm{a}$ & $15 \mathrm{~b}$ & $53 \mathrm{a}$ & $41 \mathrm{a}$ & $29 \mathrm{~b}$ \\
2 & $28 \mathrm{~b}$ & $28 \mathrm{a}$ & $22 \mathrm{~b}$ & $34 \mathrm{~b}$ & $34 \mathrm{~b}$ & $23 \mathrm{~b}$ \\
3 & $34 \mathrm{~b}$ & $32 \mathrm{a}$ & $33 \mathrm{a}$ & $41 \mathrm{a}$ & $43 \mathrm{a}$ & $35 \mathrm{a}$ \\
4 & $44 \mathrm{a}$ & $42 \mathrm{a}$ & $37 \mathrm{a}$ & $29 \mathrm{~b}$ & $32 \mathrm{~b}$ & $36 \mathrm{a}$ \\
5 & $33 \mathrm{~b}$ & $31 \mathrm{a}$ & $32 \mathrm{a}$ & $21 \mathrm{~b}$ & $25 \mathrm{~b}$ & $23 \mathrm{~b}$ \\
\hline CV (\%) & 23,0 & 34,9 & 17,1 & 23,8 & 18,3 & 24,7 \\
\hline
\end{tabular}

${ }^{1}$ Médias seguidas pela mesma letra, na coluna, não diferem entre si pelo teste de Scott-Knott a 5\% de probabilidade.

${ }^{2}$ Dados transformados em $\operatorname{arcsen}(\mathrm{x} / 100)^{1 / 2}$

No envelhecimento acelerado conduzido a $45{ }^{\circ} \mathrm{C}$ pelo método tradicional (Tabela 4), também observouse heterogeneidade no ordenamento dos lotes em níveis de vigor, verificando-se maior estresse, logo maior grau de deterioração e menor vigor. $\mathrm{O}$ período de exposição de 48 horas, indicou apenas o lote 5 no grupo de sementes de alto vigor, indicando inferioridade para os demais lotes, ao passo que o aumento de exposição para 72 horas permitiu classificar também os lotes 3 e 4 como os mais vigorosos. Por outro lado, o maior período de envelhecimento, 96 horas, não foi capaz de detectar diferenças de potencial fisiológico entre os lotes. De acordo com Spinola et al. (1998) e Marcos Filho (1999b), períodos longos de envelhecimento podem ocasionar condições muito drásticas, capazes de dificultar ou impedir a detecção de diferenças significativas de qualidade entre lotes de sementes.

Para o envelhecimento acelerado, com o uso de solução saturada de $\mathrm{NaCl}$ a $45^{\circ} \mathrm{C}$ (Tabela 4), o período de 48 horas de exposição permitiu estratificação mais evidente dos lotes, classificando sementes de alto (lote 1), médio (lote 3) e baixo vigor (lotes 2, 4 e 5). Além disso, observou-se menor coeficiente de variação para este período de exposição. Por outro lado, o procedimento com 72 horas de exposição, permitiu a classificação dos lotes apenas em duas categorias fisiológicas, de maneira semelhante ao teste de emergência de plântulas, ao apontar os lotes 1 e 3 como de alto vigor, e os lotes 2,4 e 5 como de baixo vigor. Já as condições adversas proporcionadas pelo período de 96 horas demonstraram baixa capacidade de diferenciação dos lotes ao indicar similaridade entre as sementes dos lotes 1,3 e 5 .

TABELA 4. Vigor de cinco lotes de sementes de grama-bermuda (Cynodon dactylon) após 0 teste de envelhecimento acelerado tradicional (EAT) e com uso de solução saturada de NaCl (EASS), a $45{ }^{\circ} \mathrm{C}$, em três períodos de envelhecimento ${ }^{1,2}$.

\begin{tabular}{cccccccc}
\hline \multirow{2}{*}{ Lote } & \multicolumn{3}{c}{ EAT } & & \multicolumn{3}{c}{$N$ NaCl } \\
\cline { 2 - 4 } \cline { 7 - 8 } & $48 \mathrm{~h}$ & $72 \mathrm{~h}$ & $96 \mathrm{~h}$ & & $48 \mathrm{~h}$ & $72 \mathrm{~h}$ & $96 \mathrm{~h}$ \\
\hline 1 & $24 \mathrm{~b}$ & $12 \mathrm{~b}$ & $24 \mathrm{a}$ & & $48 \mathrm{a}$ & $43 \mathrm{a}$ & $32 \mathrm{a}$ \\
2 & $20 \mathrm{~b}$ & $21 \mathrm{~b}$ & $20 \mathrm{a}$ & & $27 \mathrm{c}$ & $22 \mathrm{~b}$ & $15 \mathrm{~b}$ \\
3 & $27 \mathrm{~b}$ & $28 \mathrm{a}$ & $23 \mathrm{a}$ & & $38 \mathrm{~b}$ & $36 \mathrm{a}$ & $31 \mathrm{a}$ \\
4 & $23 \mathrm{~b}$ & $29 \mathrm{a}$ & $22 \mathrm{a}$ & & $31 \mathrm{c}$ & $28 \mathrm{~b}$ & $18 \mathrm{~b}$ \\
5 & $39 \mathrm{a}$ & $37 \mathrm{a}$ & $20 \mathrm{a}$ & & $24 \mathrm{c}$ & $21 \mathrm{~b}$ & $24 \mathrm{a}$ \\
\hline CV (\%) & 30,2 & 27,8 & 31,6 & & 15,5 & 19,1 & 29,3
\end{tabular}

${ }^{1}$ Médias seguidas pela mesma letra, na coluna, não diferem entre si pelo teste de Scott-Knott a 5\% de probabilidade.

${ }^{2}$ Dados transformados em arc sen $(\mathrm{x} / 100)^{1 / 2}$

Apesar das combinações $41{ }^{\circ} \mathrm{C} / 48 \mathrm{~h}, 41^{\circ} \mathrm{C} / 72 \mathrm{~h}$ e 45 ${ }^{\circ} \mathrm{C} / 72 \mathrm{~h}$, pelo método EASS, terem apresentado resultados semelhantes aos da emergência de plântulas, que constitui parâmetro indicador da eficiência dos testes para avaliação do potencial fisiológico de lotes de sementes (Marcos Filho, 2005), verificou-se que o poder de discriminação deste teste não foi decisivo, estratificando os lotes apenas em dois níveis de vigor. Todavia, a combinação $45{ }^{\circ} \mathrm{C} / 48 \mathrm{~h}$, demonstrou maior capacidade de diferenciação de potencial fisiológico de lotes de sementes de grama-bermuda, separando-os em classes de potencial superior, intermediário e inferior. 
Portanto, o teste de envelhecimento acelerado em sementes de grama-bermuda com solução saturada de $\mathrm{NaCl}$ na combinação $45{ }^{\circ} \mathrm{C} / 48 \mathrm{~h}$ foi o método mais adequado para classificação de lotes de sementes de grama-bermuda em diferentes níveis de vigor, podendo ser incluído em programas de controle de qualidade, auxiliando nas tomadas de decisões em diferentes etapas de produção e da utilização da semente. Cabe ressaltar que, o emprego do procedimento modificado contribui ainda para reduzir a absorção de água pela semente, acarretando em menor grau de deterioração, resultados menos drásticos e mais uniformes em relação ao envelhecimento acelerado tradicional.

\section{CONCLUSÕES}

O teste de envelhecimento acelerado com uso de solução saturada de $\mathrm{NaCl}$ permite avaliar o potencial fisiológico de sementes de grama-bermuda; a combinação 48 horas a $45^{\circ} \mathrm{C}$ é o procedimento mais adequado para detectar diferenças de vigor, possibilitando a classificação de lotes quanto ao potencial fisiológico.

\section{REFERÊNCIAS}

ARRUDA, R.L.B.; HENRIQUES, E. Gramados. São Paulo: Europa, 1995. 63p.

ÁVILA, P.F.V.; VILLELA, F.A.; ÁVILA, M.S.V. Teste de envelhecimento acelerado para avaliação do potencial fisiológico de sementes de rabanete. Revista Brasileira de Sementes, v.28, n.3, p.52-58, 2006.

BRASIL. Ministério da Agricultura e Reforma Agrária. Secretaria Nacional de Defesa Agropecuária. Departamento Nacional de Defesa Vegetal. Coordenação de Laboratório Vegetal. Regras para análise de sementes. Brasília, DF, 1992. 365p.

CARVALHO, N.M.; NAKAGAWA, J. Sementes: ciência, tecnologia e produção. 3.ed. Jaboticabal: FUNEP, 2000. $588 \mathrm{p}$.

DELOUCHE, J.C; BASKIN, C.C. Accelerated aging techniques for predicting the relative storability of seed lots. Seed Science and Technology, v.1, n.2, p.427-452, 1973.

DELOUCHE, J.C. Germinação, deterioração e vigor da semente. Revista Seed News, v.6, n.6, p.24-31, 2002.
JIANHUA, Z.; McDONALD, M.B. The saturated salt accelerated aging test for small-seeded crops. Seed Science and Technology, v.25, n.1, p.123-131, 1996.

MAGUIRE, J.D. Speed of germination: aid in selection and evaluation for seedling emergence and vigor. Crop Science, v.2, n.2, p.176-177, 1962.

MARCOS FILHO, J. Fisiologia de sementes de plantas cultivadas. Piracicaba: FEALQ. 2005, 495p.

MARCOS FILHO, J. Testes de vigor: importância e utilização. In: KRZYZANOWSKI, F.C.; VIEIRA, R.D.; FRANÇA NETO, J.B. (Ed.). Vigor de sementes: conceitos e testes. Londrina: ABRATES, 1999a. cap.1, p.1-21.

MARCOS FILHO, J. Teste de envelhecimento acelerado. In: KRZYZANOWSKI, F.C.; VIEIRA, R.D.; FRANÇA NETO, J.B. (Ed.). Vigor de sementes: conceitos e testes. Londrina: ABRATES, 1999b. cap.3, p.1-24.

PANOBIANCO, M.; MARCOS FILHO, J. Comparação entre métodos para avaliação da qualidade fisiológica de sementes de pimentão (Capsicum annuum L.). Revista Brasileira de Sementes, v.20, n.2, p.306-310, 1998.

POWELL, A.A. The controlled deterioration test. In: VERTER, H.A. VAN de. Seed vigour testing seminar, Zurich: ISTA, 1995. p.73-87.

RAMOS, N.P.; FLOR, E.P.O.; MENDONÇA, E.A.F.; MINAMI, K. Envelhecimento acelerado em sementes de rúcula (Eruca sativa L.). Revista Brasileira de Sementes, v.26, n.1, p.98-103, 2004.

RODO, A.B.; PANOBIANCO, M.; MARCOS FILHO, J. Metodologia alternativa do teste de envelhecimento acelerado para sementes de cenoura. Scientia Agricola, v. 57, n.2, p.289-292, 2000.

SPINOLA, M.C.M.; CALIARI, M.F.; MARTINS L.; TESSARIOLI NETO, J. Comparação entre métodos para avaliação do vigor de sementes de cenoura. Revista Brasileira de Sementes, v.20, n.2, p.63-67, 1998.

SOUZA, S.A.; NAKAGAWA, J.; MACHADO, C.G. Teste de envelhecimento acelerado em sementes de aveia preta. Revista Brasileira de Sementes, v.31, n.2, p.155-163, 2009.

TORRES, S.B.; MARCOS FILHO, J. Teste de envelhecimento acelerado em sementes de maxixe (Cucumis anguria L.). Revista Brasileira de Sementes, v.23, n.2, p.108-112, 2001. 